\title{
Right ventricle free wall longitudinal strain for diagnosis of right ventricular infarction in inferior wall myocardial infarction
}

\author{
Wei-Chieh Lee ${ }^{1,2}$
}

Published online: 20 July 2021

(c) The Author(s), under exclusive licence to Springer Nature B.V. 2021

We greatly enjoyed reading the recently published article by Ahmed et al. [1]. The authors reported that a cutoff value of right ventricle free wall longitudinal strain (RVFWLS) $\leq-20.5 \%$ had $88 \%$ sensitivity and $33 \%$ specificity for RV infarction diagnosis in patients with acute inferior ST-segment elevation myocardial infarction (STEMI). This was a cross-sectional observational study and enrolled 100 consecutive patients with acute inferior STEMI. According to the criteria of RV infarction by electrocardiography, only $25 \%$ of patients met the criteria. After excluding patients whose target lesion was not at the right coronary artery (RCA), 42 patients had proximal RCA lesions, and 31 patients had distal RCA lesions. Many echocardiographic methods of evaluating RV function, including fractional area change, tricuspid annular peak systolic excursion (TAPSE), isovolumetric contraction time, isovolumetric relaxation time, ejection time, myocardial performance index, right ventricle global longitudinal strain (RV-GLS), and RVFWLS, were compared between patients with proximal and distal lesions. Only RV-FWLS showed a significant difference between groups and had predictive value.

Prior studies presented a higher incidence of mortality and complications in inferior STEMI with RV infarction. The reasons were mainly related to the increased risk of lifethreatening arrhythmias. Therefore, proper monitoring and appropriate antiarrhythmic treatment play a key role in the survival of patients during a critical phase. Previous studies

This comment refers to the article available online at https://doi. org/10.1007/s10554-021-02311-2.

\section{Wei-Chieh Lee}

leeweichieh@yahoo.com.tw

1 Institute of Clinical Medicine, College of Medicine, National Cheng Kung University, Tainan, Taiwan

2 Division of Cardiology, Department of Internal Medicine, Kaohsiung Chang Gung Memorial Hospital, Chang Gung University College of Medicine, No, 123, Ta Pei Road, Niao Sung District, 833 Kaohsiung, Taiwan showed that RV-GLS could predict proximal RCA stenosis and early mortality in patients with acute inferior STEMI. In clinical practice, TAPSE is easily performed, but difficultly estimates the global function of a complex structure from a single-segment analysis and is easily affected by left ventricular dysfunction. RV-GLS and RV-FWLS evaluate $\mathrm{RV}$ function for many cardiovascular diseases, including dilated, hypertrophic, ischemic, infiltrated, and arrhythmogenic right ventricular cardiomyopathy, by echocardiography or cardiac magnetic resonance imaging. In this study, RV-FWLS $\leq-20.5 \%$ provided high sensitivity for the diagnosis of RV infarction in patients with acute inferior STEMI.

In patients with HF, RV-GLS and RV-FWLS are sensitive tools to evaluate RV function and predict prognosis in HF patients [2]. RV-GLS might be influenced by left ventricular (LV) dysfunction because the interventricular septum is an integral part of the LV. Therefore, RV-FWLS better predicts outcomes than RV-GLS. In patients with significant tricuspid regurgitation, impaired RV-FLS identifies higher rates of RV dysfunction and is associated with worse outcomes beyond conventional echocardiographic parameters of RV systolic function. Ahmed et al. reported that RV-FWLS $\leq-20.5 \%$ provided a high sensitivity for the diagnosis of RV infarction in patients with acute inferior STEMI. In addition, ST-segment elevation in the V4R lead and proximal culprit lesions in the RCA were predictors of impaired RV-FWLS.

Therefore, RV-FWLS plays an important role in evaluating RV performance and may be independent of LV performance. Early detection of RV dysfunction may improve the outcomes of acute inferior STEMI with RV infarction.

\section{Acknowledgements None.}

Funding None. 


\section{Declarations}

Conflict of interest The authors declare that they have no conflict of interest.

\section{References}

1. Ahmed TAN, Abdel-Rahman EG, Helmy HA, Hasan-Ali H, El-Naggar HM (2021) Role of 2-dimensional speckle tracking echocardiography in diagnosis of right ventricular involvement in patients with inferior wall myocardial infarction undergoing primary percutaneous coronary intervention. Int J Cardiovasc Imaging. https://doi.org/10.1007/s10554-021-02311-2

2. Carluccio E, Biagioli P, Lauciello R, Zuchi C, Mengoni A, Bardelli G, Alunni G, Gronda EG, Ambrosio G (2019) Superior prognostic value of right ventricular free wall compared to global longitudinal strain in patients with heart failure. J Am Soc Echocardiogr 32:836-844.e1

Publisher's Note Springer Nature remains neutral with regard to jurisdictional claims in published maps and institutional affiliations. 